\title{
Liquid and Solid Carbon Particles in Cool White Dwarf Atmospheres
}

\section{VICTOR ZUBKO ${ }^{1,2}$}

\author{
${ }^{1}$ Institute of Astronomy, N. Copernicus University, Toruń, Poland \\ 2 on leave from the Main Astronomical Observatory, NAS, Kiev, Ukraine
}

\begin{abstract}
About ten years ago Zhilyaev \& Zubko (1983, PAZh, 9, 227; 1984, ApESSS, $105,99)$ theoretically predicted that carbon in the condensed form may be present in the atmospheres of cool white dwarfs. In particular, it was shown by the model-atmosphere technique that models with effective temperature $T_{\text {eff }} \leq 6000 \mathrm{~K}$, gravitation $g=10^{8} \mathrm{~cm} \mathrm{~s}^{-2}$, and chemical abundances $\mathrm{H} / \mathrm{He} \leq$ $10^{-5}, \mathrm{C} / \mathrm{He} \leq 0.001$ and $\mathrm{C} / \mathrm{O}>1$ may contain both liquid carbon droplets and solid dust grains, forming a stable layer inside the atmosphere. Now there are opportunities to explore the problem from two directions. The first one concerns the observational search for signs of condensed carbon in the spectra of cool white dwarfs which may be obtained with powerful modern groundand space-based facilities. In any case, the first observations of white dwarfs with the Hubble Space Telescope (e.g. Shipman et al. 1995, AJ, 109, 1231) are very promising. The second line of attack is the calculation of refined model atmospheres of cool white dwarfs as possible carriers of carbon condensate, on the basis of relevant model-atmosphere codes (e.g. Bergeron, Saumon \& Wesemael 1995, ApJ, 443, 764; Aslan \& Bues, this volume) supplemented by sophisticated calculations of the thermochemical and ionization equilibrium in complicated molecular systems (Schmidt, Bergeron \& Fegley 1995, ApJ, 443, 274). However, the physical description of condensate particles in the framework of the thermodynamical approach used in early studies seems overly simplified. We propose an improved microphysical model of the carbon condensate layer in a white dwarf atmosphere based on the stationary solutions of an appropriate kinetic equation. The most important physical processes to be taken into account in modelling a condensate layer are the nucleation, growth-evaporation and sedimentation of condensate particles. The coagulation and diffusion of particles through a condensate layer are inefficient and may be neglected. Estimates show that micronic particles prevail in a condensate layer and that the solid carbon particles should be amorphous rather than crystalline. A condensate layer may become optically thick at visual wavelengths as soon as the fraction of condensed carbon exceeds $1 \%$.

It is a pleasure to thank the SOC of IAU Symposium 177, the International Astronomical Union, and N. Copernicus University (Torun, Poland) for financial support. This paper has appeared in MNRAS, 287, 583, 1997.
\end{abstract}

